\title{
ANYSEP: A Program Package for Store Separation Analysis
}

\author{
Seungsoo Lee*, Keeyoung Choi* and Jin Yeon Cho** \\ Dept. of Aerospace Engineering, Inha Univeristy, Korea, 402-751
}

Sangho Kim ${ }^{* * *}$, Jaesoo Hyun***, Namgyun Kim**** and Jong Kook Lee****

Agency for Defense Development, Daejoen, Korea, 305-600

\begin{abstract}
A program package for store separation analysis, SAFESEP, has been developed. The package includes CTS system software, 3-Dimensional visualization software, off-line store trajectory software and aerodynamic database software. In this paper, we describe the functions and the capabilities of the store separation analysis program package.
\end{abstract}

\section{Introduction}

Unlike civil aircraft, military aircraft release stores such as bombs, missiles or external fuel tanks on purpose. The analysis methods for the trajectory of the store are known to be quite complicated mainly due to the difficulties in computing the interference aerodynamics of the stores in presence of the aircraft.[1] Therefore, the analysis has to rely on experimental techniques.

The Captive Trajectory Support(CTS) system is a model support which can move stores such as bombs or missiles along a predicted trajectory and can simultaneously measure the aerodynamic forces and moments acting on the stores. The CTS can be used either trajectory mode or grid test mode. In the trajectory mode, the trajectory of the store is computed simultaneously with the measured aerodynamics as the store moves inside the wind tunnel as accordance to the solution of equations of motion of the store. Therefore, the store moves along the computed trajectory as if it separates from the aircraft. For the grid test mode, the interference flow field is measured with CTS at the predetermined grid points and the aerodynamic forces and moments are converted into a grid database. Then, the trajectory is computed with the grid database with an off-line 6 degree-of-freedom(DOF) simulation program.

$\mathrm{ADD}$ (Agency for Defense Development) is planning to build a CTS system for their tri-sonic tunnel. As a preliminary study, it was decided to develop an operating system for the CTS system. A team of Inha University and ADD had developed a software package which consists of a CTS system software, an off-line 6-DOF simulation software, a grid database software and a 3-D visualization software. In this paper, we describe the functions and capabilities of the trajectory analysis program package. Also, a couple of the simulation examples were performed to show the usefulness and the validity of the package

\section{The Package}

As stated earlier, the CTS system can be operated in either the trajectory mode or the grid test mode. The trajectory mode utilizes the interactive operation of a computer, a six DOF remotely controlled mechanism and a wind tunnel. The trajectory is calculated using the currently measured

* Professor

E-mail : slee@inha.ac.kr

Tel : 032-860-7358

FAX : 032-865-5401

** Associate Professor

*** Principal Researcher

**** Senior Researcher 


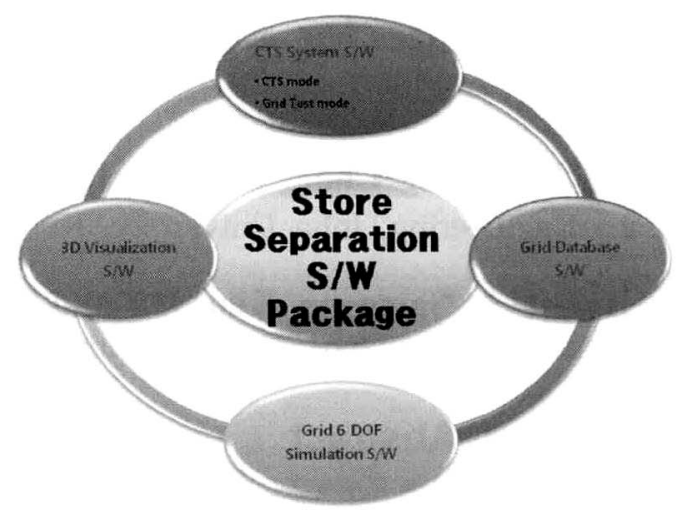

Fig. 1. CTS system software package

store aerodynamic loads in the solution of the equations of motion, the results of each step in the calculation being used to reposition the store relative to the aircraft model in the wind tunnel. The trajectory mode is quite expensive to test. Therefore, most of the CTS testing are done with the grid test mode. First, the grid test mode starts the measuring the interference aerodynamic loads at the predetermined points near the model aircraft. This aerodynamic loads and the free-stream aerodynamic database of the store are used generate the interference database known as the grid database. The 6-DOF simulation program computes the trajectory of the store using the grid database and the free-stream database.

Figure 1 depicts the structure of the software package and the relations between the softwares. The CTS system software is an operating system for the CTS system, while the off-line 6-DOF simulation software is an engineering trajectory prediction program which uses the grid database generated by the aerodynamic database software from the grid test. The 3-D visualization software display what might happen inside the wind tunnel, including the aircraft, the store and the CTS rig.

\section{CTS System Software}

The main modules of the CTS system software are GUI module, aero module, trajectory module, inverse kinematic module and collision avoidance module.

\section{A. Graphic user interface(GUI) module}

The CTS system software has a graphic user interface which allows engineers to set up a test easily. Figure 2 shows test setup by merging an aircraft, a pylon and a store selected from the configuration databases which are defined earlier. The combined configuration will be used in the $3-\mathrm{D}$ visualization software as well as in the collision avoidance module.

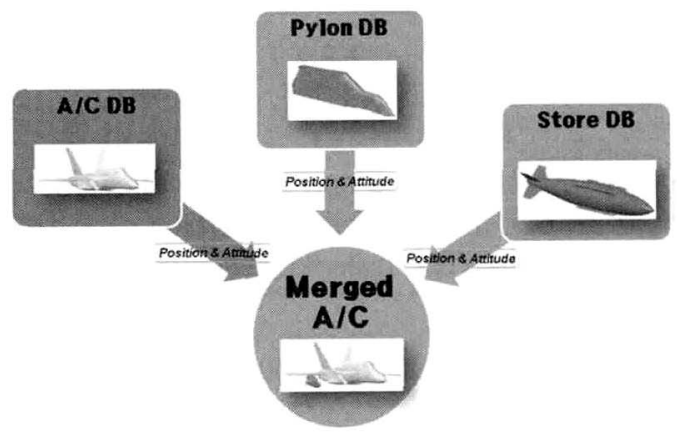

Fig. 2. Test setup from configuration databases 

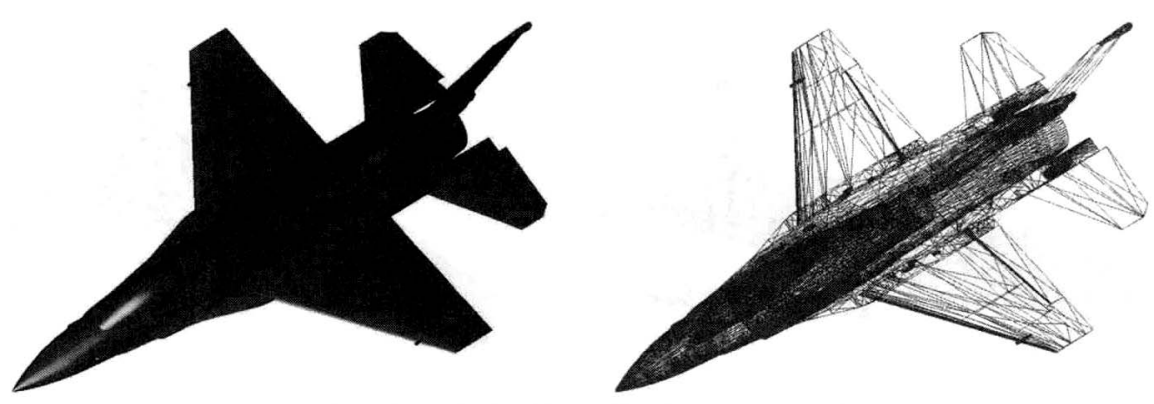

Fig. 3. CAD surface and STL

The configuration databases consist of aircraft database, pylon database, rack database, store database, aircraft support database, CTS rig database and balance database. Each database includes the $\mathrm{CAD}$ data and the engineering characteristics of the configuration. The $\mathrm{CAD}$ format used in the software is STL(Standard Tessellation Language), which can be generated by any commercial $\mathrm{CAD}$ programs. Figure 3 shows the CAD surface of an combat aircraft and its SLT representation.

\section{B. Aero module}

The aero module not only converts the balance signal to aerodynamic forces and moments but also computes and removes weight tare from the balance signal.

\section{Trajectory module}

The trajectory module computes the trajectories of stores from the aerodynamic forces moments with or without constraints. The store can be release based on one of three standard constrained release scenarios; pivot motion, rail launch and ejector plane motion. Usually fuel tanks are released with the pivot motion. Rail launch motion is used for a missile launch. The ejector plane motion restricts store motion only to the ejector plane during the ejector strokes. This option is typically used for stores ejected from suspension equipment. Stores may be release from an aircraft other than in straight level flight, such as level acceleration, steady roll-out, pull-up or push-over.

In order to reduce the possible collision of stores with the mother aircraft, ejectors are used during the release to push the stores out of the interference flow field. Missile trajectory computation requires the thrust effect on the missile motion. Therefore, the ejector forces and the thrust are modeled in trajectory computation. Moreover, autopilot option is added in the aerodynamic forces and moments to account for the deflection of control surfaces during the separation of a smart bomb.

The Newmark-beta method is used to integrate the equations of motion for the constrained releases and the unconstrained releases. The 4 stage Runge-Kutta method and a predictor-corrector method which couples Adams Moulton method and Adams Bashforth method can be used for the unconstrained releases.

\section{Inverse kinematics module}

The inverse kinematics module determines the command inputs for servo actuators from the displacements and the orientations of the store which are computed with the trajectory module. Since the rig mechanism for ADD's tunnel has not been decided, a general inverse kinematics for 6 DOF rig mechanism is devised. Only restriction on the rig mechanism is that the translation motions precede the rotation motions.

\section{E. Collision avoidance module}

During the trajectory simulation, the collision between the store mode and the aircraft model or the CTS rig may happen. The collision avoidance module prevents this from happening by examining the minimum distance between the store and the rest of the models including the aircraft, the aircraft support, the CTS rig and the wind tunnel walls. In order to reduce the time to compute the minimum 

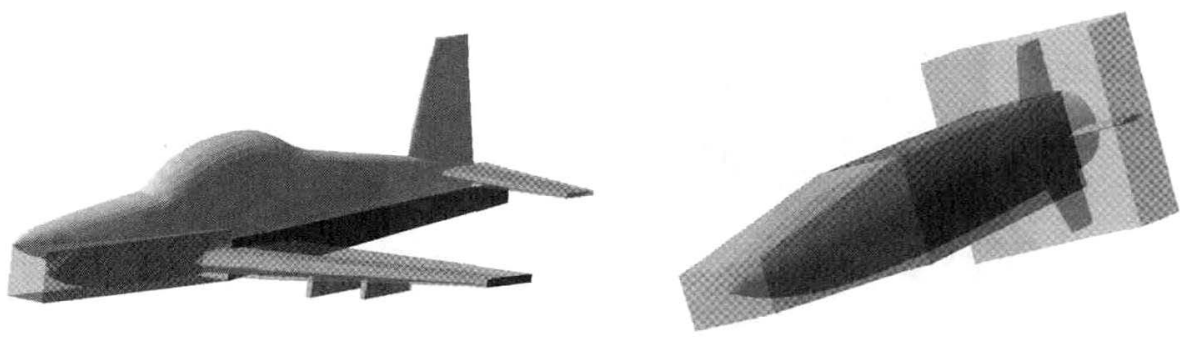

Fig. 4. AABB's over aircraft and store

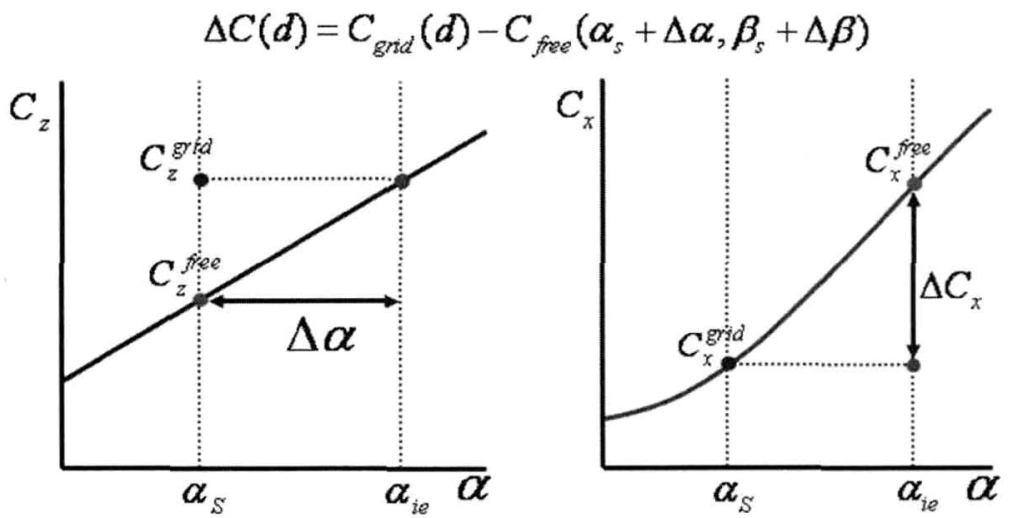

Fig. 5. Interference effects, flow angularity method

distance, AABB's(Axis Align Bounding Boxes) are generated over all models and the minimum distance is approximately estimated by the minimum distance between AABB's. Figure 4 shows AABB's over the aircraft and the store. Note that the AABB's cover only the critical region of the aircraft, where the collision might be expected to happen.

\section{Grid Database Software}

The grid database software generates the interference aerodynamic database which will be used for the 6-DOF simulation software. The basic idea of the flow angularity method is to represent the inference effects of the aircraft with local flow angle changes which are approximately assumed to be functions of the distance from the aircraft only. The angle of attack and the angle of sideslip are computed from the grid test data with the free-stream test data of the stores. Figure 5 depicts the procedure of computing the interference effects. The detailed methodology can be found in Ref. [2].

\section{6-DOF Simulation Software}

The 6-DOF simulation software is an engineering trajectory generation program, where the interference aerodynamic forces and moments are estimated from the grid database and the free-stream database of a store. The flow angularities are interpolated from the grid database based on the distance from the captive position. These angles are added to the angle of attack and the angle of sideslip of the store. The residual coefficients are also added to estimate the aerodynamic forces and moments under the influence of the aircraft. Unsteady damping terms are added to the moment coefficients to compensate the quasi-steady nature of wind tunnel testing. The basic structure of the $6-\mathrm{DOF}$ simulation software shares with the CTS system software so that most of options related to the trajectory computation are also available. 


\section{3-D Visualization Software}

The main function of the 3-D visualization software is to display what is happening inside the wind tunnel during the wind tunnel testing. Also, it can be used as a post processor for the 6-DOF simulation software. Figure 6 shows a snap shot of output window of the 3-D visualization software. A F/A-18C aircraft, a model support, a JDAM(Joint Direct Attack Munitions) model and a CTS rig are displayed in the figure. Note that the partial view of the wind tunnel walls can be seen in the figure.

\section{Integration with Wind Tunnel}

The CTS system software developed in this study will be installed into ADD's tri-sonic wind tunnel with the CTS system. Figure 7 depicts the current framework of the CTS system software and the final framework of the CTS system software after the integration with the wind tunnel. Note that a virtual wind tunnel is designed so that it can be replaced with the real wind tunnel without major modification. In the final framework, the CTS system software acts as an operating system for the CTS system which not only generates the trajectory of the store, and the servo command for the CTS rig but also manages the data flow with a middleware which handles solely the data management.

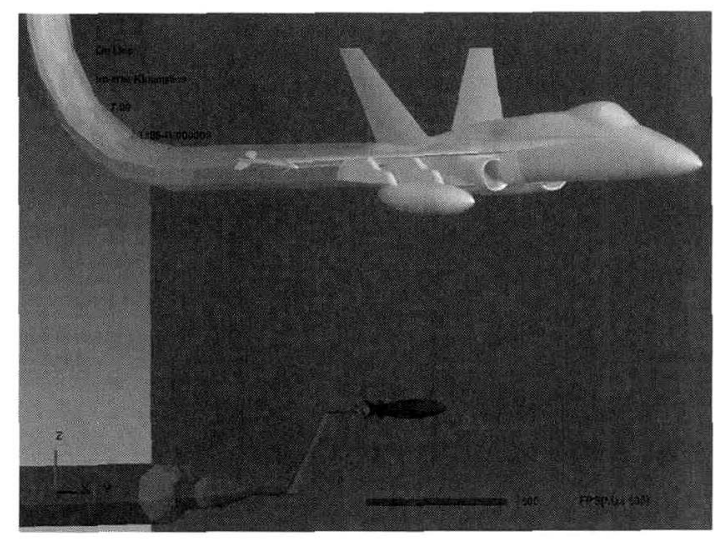

Fig. 6. 3-D Visualization software
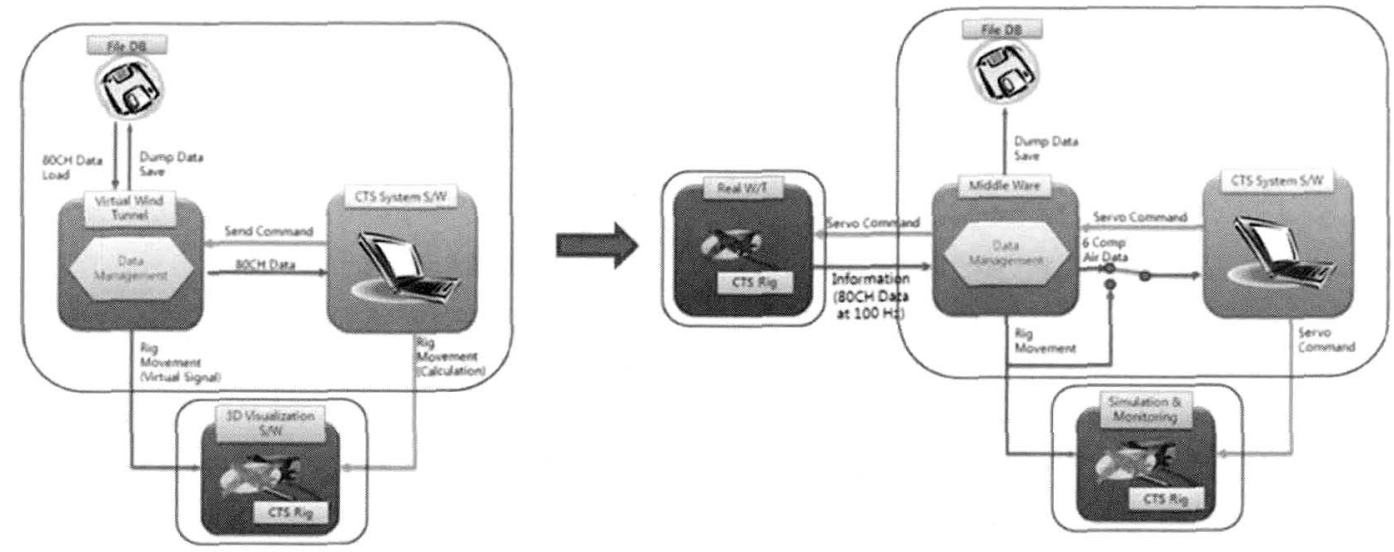

Fig. 7. Integration with wind tunnel 


\section{Computational Examples}

Figure 8 shows the trajectories of JDAM separating from F/A-18C. The flight Mach number is 0.962 , the angle of attack is $0.46^{\circ}$ and the altitude of the simulation is $6,332 \mathrm{ft}$. F/A-18C is in $43^{\circ}$ dive when it releases JDAM. Computed trajectories with the CTS system software are compared with those of MSAP(Multi-body Separation Analysis Program)[3], which is a separation analysis code based on CFD. The trajectories with the CTS system software are obtained with the aerodynamic forces and moments computed by MSAP. The resulting trajectories, therefore, are identical to those with MSAP as can be seen in the figure.

The next example is the trajectory simulation of an external bomb from a combat aircraft. The flight Mach number is 0.85 , the angle of attack is $1.3^{\circ}$ and the altitude is assumed to be $3,000 \mathrm{ft}$. The aircraft is in straight level flight. The store is ejected from the aircraft with two ejectors. The grid database is constructed from the grid test while the free-stream database of the store is obtained with the same model with the aircraft moved far away from the store model. The trajectories computed with the 6-DOF simulation software are compared with the trajectories of the CTS test for the same flight condition in Fig. 9. As can be seen in the figure, the results of the 6-DOF simulation match well with those of the CTS test except the roll angle, which is known to be difficult to predict.[4]
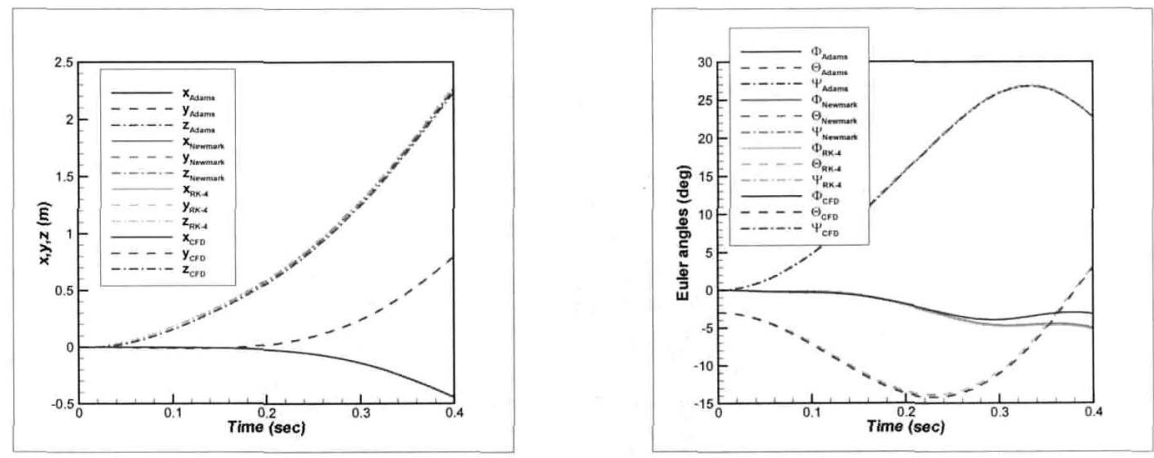

Fig. 8. Comparisons of trajectories of JDAM
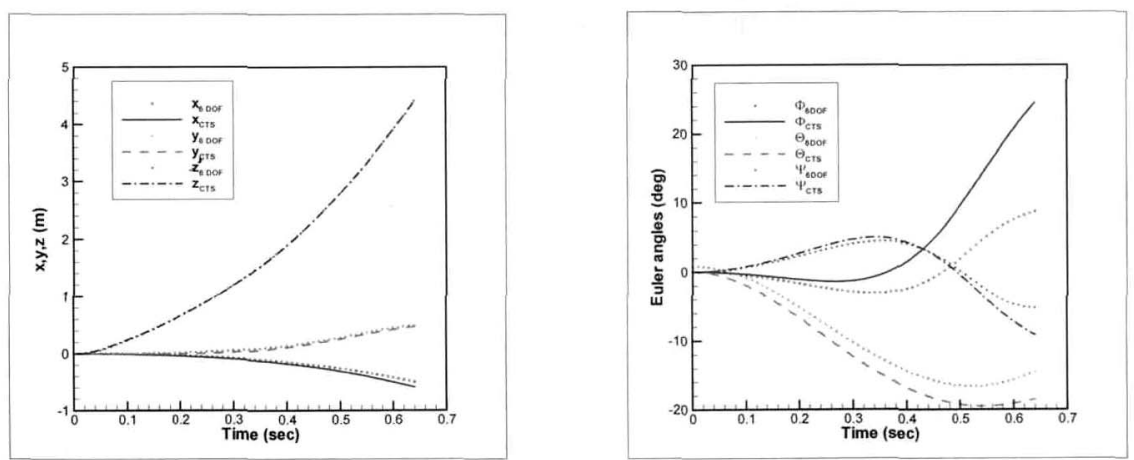

Fig. 9. Comparisons of trajecotries of store

\section{Summary}

In this study, we report the successful development a software package for store separation analysis. The package covers all the aspect of engineering analysis of store separation using wind tunnel testing. The validity of the package is shown by comparing the trajectory results with that of other method. The CTS system software will be installed in ADD's tri-sonic wind tunnel in near future. 


\section{Acknowledgements}

The work has been supported by the Agency for Defense Development.

\section{References}

1. Anonymous, “Aircraft/Stores Compatibility: Systems Engineering Data Requirements and Test Procedure", MIL-HDBK-1763, Department of Defense, 1998.

2. S. Lee, "Application of MSAP to JDAM Separation from an F/A-18C Aircraft", Journal of Computational Fluids Engineering, Vol.9, No.1, 2004.

3. B.K. Kim, S. Lee, S.J. Kim, I.M. Kang, and M.S. Kim, "Separation Analysis of a Store with Deployable Wings", Vol. 35, No. 5, Journal of the Korean Society for Aeronautical and Space

4. A. Cenko, D. Gowanlock, M. Lutton, and M. Tutty, "F/A-18C/JDAM Applied Computational Fluid Dynamics Chanllenge II Results", AIAA-2000-795, the 38th Aerospace Sciences Meeting, 2000.

5. D.T. Veazey, "Current AEDC Weapons Separation Testing and Analysis to Support Flight Testing”, AIAA paper 2004-6847, USAF Development Test and Evaluation Summit, Nov. 2004.

6. J.B. Carman, Jr. "Store Separation Testing Techniques at the Arnold Engineering Development Center”, Vol. I-IV, 1980. 